\title{
Auf dem Weg zu einer wissenschaftlichen Erkenntnis
}

\section{Empfehlungen zum Anfertigen von Bachelor- und Masterarbeiten}

Beim Anfertigen einer wissenschaftlichen Arbeit, die nach drei bzw. sechs Monaten zur Prüfung eingereicht wird, steht der Autor auf dem Weg zur wissenschaftlichen Erkenntnis zunächst mindestens vor drei Fragen: Wie findet er eine bearbeitbare Aufgabenstellung? Auf welchem Weg gelangt er zur Lösung der Aufgabe, zu einer Erkenntnis? Wie sichert er die Gültigkeit dieser Erkenntnis ab und welche Forschungsperspektive kann er aufzeigen? Dazu werden Standpunkte und Erfahrungen zur Diskussion gestellt. Schemata sollen das Behalten und Reproduzieren unterstützen, Beispiele das Vorgehen veranschaulichen.

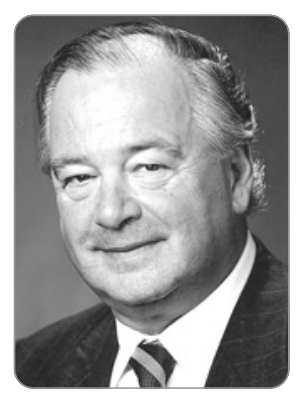

Prof. Dr. Günter Lehmann

ist Vizepräsident des Europäischen Instituts für postgraduale Bildung an der TU Dresden (EIPOS) sowie Dozent an der Dresden International University (DIU). Bevorzugte Forschungsgebiete: Techniken wissenschaftlichen Arbeitens, Ingenieurpädagogik.

Stichwörter: Themenfindung, Forschungsfrage, Hypothese, These, Feldforschung, Materialforschung, interne Validität

Wissenschaftliche Arbeiten mit Prüfungscharakter tragen zur Erweiterung des Erkenntnisstandes in Theorie und Praxis mit dem Anspruch auf Allgemeingültigkeit bei. Wesentlich ist dabei, dass vom vorhandenen Wissensstand ausgegangen wird und der Erkenntnisgewinn durch Übertragen auf neue Phänomene, Zusammenhänge oder Bereiche erzielt wird. Dazu gehören auch das Feststellen von Erkenntniswidersprüchen oder das Aufdecken von Scheinkorrelationen bzw. überholter Annahmen und Vorgehensweisen. Erkennen signalisiert Einsicht in den Gegenstand der Untersuchung. Diese Einsicht ist im Unterschied zum einfachen Kennen durch Verstehen gekennzeichnet und besitzt den Charakter des Neuen. Immer dann, wen erstmalig ein Zusammenhang oder eine Problemlösung unter definierten Bedin- gungen gefunden wurde, handelt es sich um eine Erkenntnis. So gesehen ist das Benennen von Vermutungen, Ahnungen oder auch Irrtümern an sich noch keine Erkenntnis.

Langjährige Erfahrungen in der Betreuung und Begutachtung von Bachelor-, Master-, Magister- und Diplomarbeiten verweisen neben der Einhaltung von Konventionen auf drei Erfolgsfaktoren auf dem Weg zum wissenschaftlichen Ergebnis.

\section{Finden einer bearbeitbaren Aufgaben- bzw. \\ Fragestellung}

Eine wichtige Quelle für Arbeitsthemen ergibt sich aus der persönlichen Lebens- und Arbeitswelt des Autors. Die Themenlisten der Hochschulinstitute und ihrer Forschungscluster bieten darüber hinaus zahlreiche Anregungen. In Abb. 1 sind weitere Möglichkeiten eröffnet.

Allen auf diesem Wege gefundenen Arbeitsthemen ist allerdings in der Regel gemeinsam, dass sie bei sorgfältigem Nachprüfen weder dem inhaltlichen Anspruch an die jeweilige Prüfungsarbeit noch den zeitlichen Bedingungen für die Bearbeitung entsprechen. In der Regel besitzen sie den Charakter übergreifender Zielstellungen, zu deren Erfüllung die Arbeit nur einen Einzelbeitrag leisten kann. Diesen Beitrag schlüssig aus mehreren Möglichkeiten auszuwählen und in eine bearbeitbare Aufgaben- bzw. Fragestellung zu überführen, ist ein erster wichtiger Schritt auf dem Weg zum Erfolg.

Dazu wird die schrittweise Beantwortung der folgenden Fragen empfohlen: 


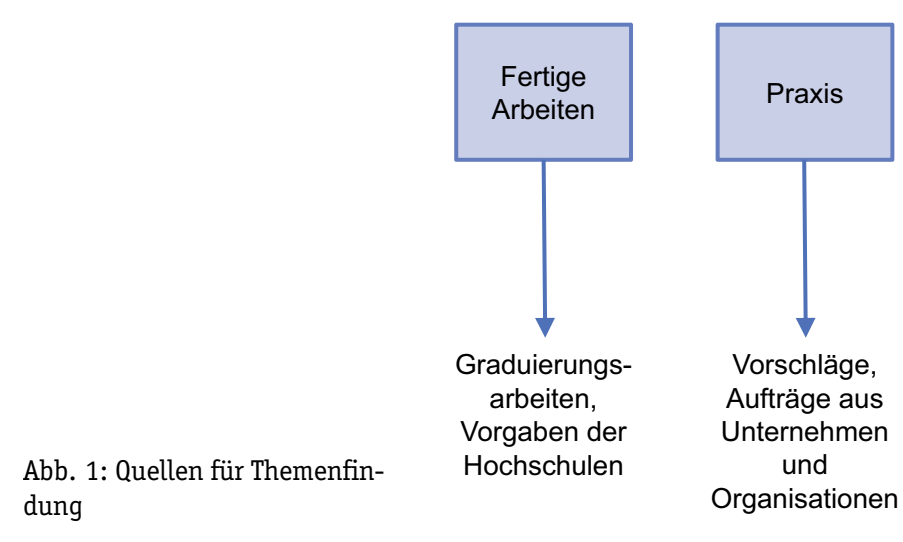

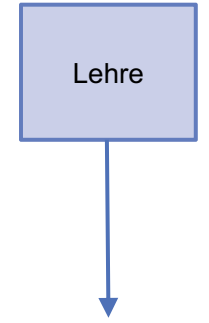

Ideenquelle

Lehrveranstal-

tung, siehe

Themen-

recherche

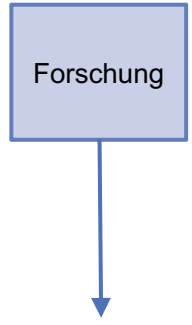

Fo-Studien,

Fachliteratur,

insb. Fachzeit-

schriften

(„Desiderato“)

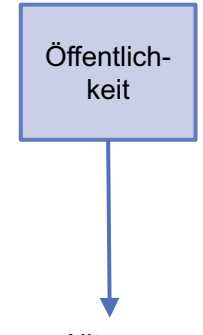

Alltagserfahrungen

(z. B. Störungen),

Tagespresse,

Medien, etc.
Schritt 1: Wozu will ich mit meiner wissenschaftlichen Arbeit einen Beitrag leisten?

- Das ist die Frage nach einem übergreifenden Ziel (keinesfalls mit dem Thema identisch).

Schritt 2: Welche Lösungsmöglichkeiten gibt es für das Erreichen des übergreifenden Ziels?

- Das führt zu mehreren Fragestellungen (nicht bei einer Fragestellung stehen bleiben).

Schritt 3: Welche von diesen Fragestellungen sind bereits (weitgehend) beantwortet?

- Das erfahre ich im Ergebnis der Literaturrecherche und der Konsultation von Dozenten und anderen Fachleuten.

Schritt 4: Welche der noch weitgehend unbeantworteten Fragen untersuche ich im Ergebnis einer Auswahl?

- Wesentliche Auswahlkriterien: Bezug zu einem Fach des Studienganges, eigenes Interesse, Zugangsmöglichkeiten zu notwendigen Daten, Neuigkeits- bzw. Schwierigkeitsgrad, Verwertungsinteressen Dritter (z. B. Promoter).
- Damit bestimme ich eine Aufgabenstellung als Arbeitsthema für meinen Beitrag zum Erreichen des übergreifenden Ziels.

Schritt 5: Welche der unbeantworteten Frage untersuche ich im Ergebnis der Auswahl nicht?

- Damit verweise ich zugleich auf Themen für künftige wissenschaftliche Arbeiten (Forschungsperspektive).

Schritt 6: Wie kann ich die gewählte Aufgabenstellung (Arbeitsthema) so präzisieren, dass sie unter den gegebenen Prüfungsbedingungen (z. B. wissenschaftlicher Anspruch, verfügbare Bearbeitungszeit) als Thema meiner wissenschaftlichen Arbeit bearbeitbar wird oder bleibt?

- Prüfkriterien: Begriffliche Eingrenzung, örtliche und/ oder räumliche Einschränkung, institutionelle Eingrenzung, zeitliche Begrenzung, methodisches Vorgehen, spezifizierte Aspekte.

Abb. 2 illustriert ein Beispiel für das Ableiten einer Aufgabenstellung entsprechend der vorgestellten Schrittfolge.
Abb. 2: Beispiel für eine Schrittfolge zur Themenfindung (Hindernisrecherche)
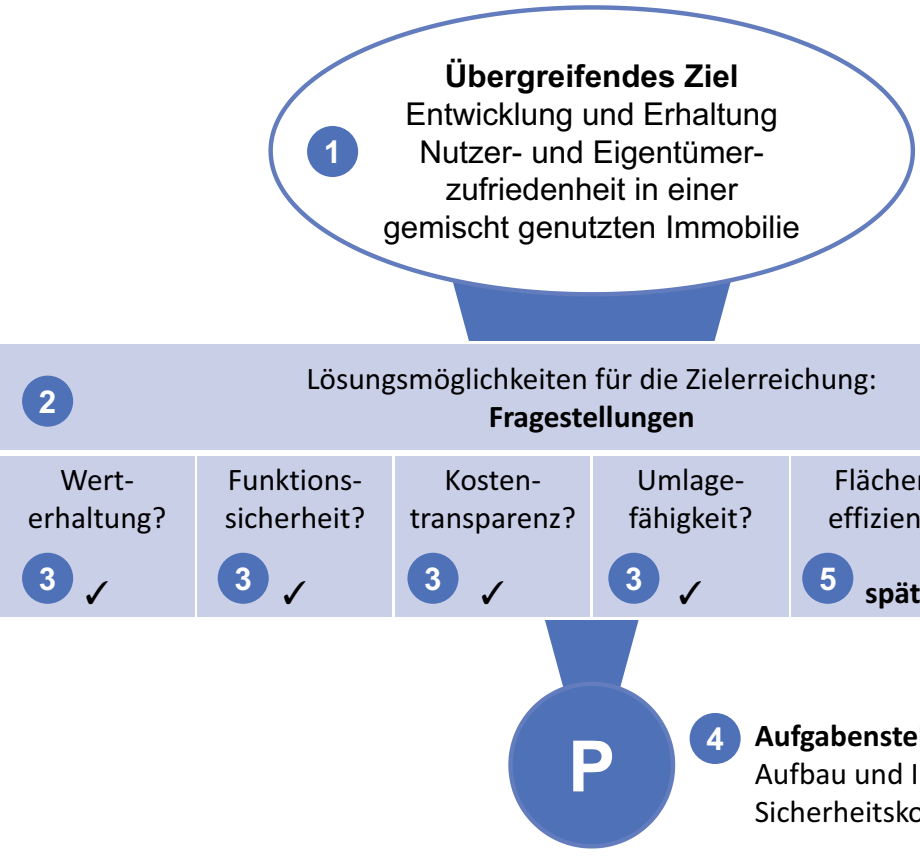

Legende:

P - Person des Verfassers

2. Schritte im Denkschema
6 Thema:

Analyse der nächtlichen Gefährdungslage im Außenbereich der Immobilie
Aufgabenstellung (Arbeitsthema):

Aufbau und Implementierung eines Sicherheitskonzeptes der Immobilie

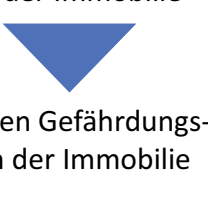


Ein Blick auf die im Schritt 6 genannten Prüfkriterien zeigt, dass ihr Einsatz jeweils die stärkere Konkretisierung oder auch Verallgemeinerung des Themas in Abhängigkeit vom Typ der wissenschaftlichen Arbeit (Haus-, Bacheloroder Masterarbeit) ermöglicht. Hinter dem im Beispiel formulierten Thema stehen beispielsweise folgende Forschungsfragen: „Was kennzeichnet die nächtliche ...?” oder „Welche Faktoren bestimmen die nächtliche ...?”.

Neben der soeben beschriebenen Hindernisrecherche lässt sich die Schrittfolge auch über die bewährten Instrumente der Ideengrafik, der Zielbaumtechnik oder der Typisierung (W-Fragestellungen oder Vorgehensweisen) umsetzen (vgl. Lehmann, 2017, S. 18 ff.). In allen Fällen geht es immer um das Finden mehrerer Möglichkeiten für das Erreichen eines übergreifenden Ziels, die Auswahl einer Aufgabenstellung als Arbeitsthema für den eigenen Beitrag und das Präzisieren der Aufgabenstellung in Abhängigkeit von den Prüfungsbedingungen für die wissenschaftliche Arbeit.

\section{Beschreiten eines nachvollziehbaren Weges zu Lösung der Aufgabe, zum Erkenntniszuwachs}

Das gefundene Thema impliziert eine oder mehrere Forschungsfragen, deren Beantwortung den Kern der folgenden Untersuchung bildet. Die Forschungsfrage muss realistisch bearbeitbar, insbesondere in der vorgegebenen Zeit mit der vorhandenen Fach- und Methodenkompetenz des Verfassers beantwortbar sein (vgl. Klein. 2017, S. 112). Hinter der Forschungsfrage steht in der Regel eine Annahme, d. h. eine Hypothese über die Existenz eines bestimmten Phänomens. Eine wissenschaftliche Hypothese besitzt einen eindeutigen Informationsgehalt und ist empirisch überprüfbar (vgl. Trimmel, 2009, S. 46). Im Unterschied zur existenziellen Hypothese erschöpft sie sich allerdings nicht in der Annahmen, dass z. B. "A $\mathrm{A}$ " existiert, sondern dass " $\mathrm{A}$ " mit " $B$ " oder " $C$ " oder mit beiden im Zusammenhang („wenn-dann“ oder „je-desto“) steht. Dieser Zusammenhang darf sich nicht auf einen Einzelfall reduzieren, sondern muss für eine Klasse von Fällen verallgemeinerbar sein. Außerdem ist sie falsifizierbar, kann also stets auch wiederlegt werden (vgl. Karmasin, Ribing, 2013, S. 93). So erfüllt beispielsweise die Aussage "Alle Schwäne sind weiß" allen Anforderungen an eine wissenschaftliche Hypothese. Die Aussage „Es gibt weiße Schwäne“ erfüllt dagegen diesen Anspruch nicht.

Mit Blick in die einschlägige wissenschaftliche Literatur, also auf dem Weg der Materialforschung wird nun geprüft, ob der in der Hypothese angenommene Zusammenhang bereits im gleichen oder in anderen Bereichen untersucht und diagnostiziert wurde. Lässt das Literaturstudium oder das Gespräch mit Fachleuten erkennen, dass kein direkter Zusammenhang, keine messbare Beziehung zwischen " $\mathrm{A}^{\prime}$ und " $B$ " besteht, ist die bisherige Hypothese zu variieren, zu verändern oder gar zu verwerfen. So kann das Studium der wissenschaftlichen Literatur auch zu neuen Hypothesen führen.

In der nun folgenden Feldforschung im weiteren Sinne (unter Einbeziehung des Laboratriums und des nichtwissenschaftlichen Schriftgutes) wird die Gültigkeit des angenommenen Zusammenhangs zwischen " $\mathrm{A}$ “ und „ $\mathrm{B}$ “ für den Bereich " $\mathrm{X}^{\prime \prime}$ verifiziert (bestätigt) oder falsifiziert (widerlegt). Typische Instrumente hierfür sind die Befragung, das Interview, die Beobachtung, das Experiment oder die Inhaltsanalyse. Im Ergebnis wird der Erkenntniszuwachs mit exakter Angabe des Gültigkeitsumfangs als Behauptung, also als These formuliert.

Für Graduierungsarbeiten ist der Nachweise von Schlussfolgerungen aus den erreichten Ergebnissen unverzicht-

\begin{tabular}{|c|c|c|c|c|}
\hline \multicolumn{5}{|c|}{ Graduierungsarbeiten } \\
\hline \multicolumn{4}{|c|}{ Projektarbeiten } & \multirow[b]{2}{*}{ Umsetzung } \\
\hline $\begin{array}{l}\text { subjektive } \\
\text { Sicht }\end{array}$ & $\begin{array}{l}\text { objektiver } \\
\text { Befund }\end{array}$ & $\begin{array}{l}\text { Verifikation } \\
\text { Falsifikation }\end{array}$ & Ergebnis & \\
\hline+ & 1 & 1 & 3 & $+1=4$ \\
\hline $\begin{array}{l}\text { Eigene } \\
\text { Erkennt } \\
\text { nisse und } \\
\text { Erfahrungen } \\
\text { Forschungs- } \\
\text { frage }\end{array}$ & $\begin{array}{c}\text { Ergebnisse } \\
\text { der Materiat } \\
\text { forschung } \\
\text { (Sekundäf } \\
\text { material) }\end{array}$ & $\begin{array}{l}\text { Ergebnisse } \\
\text { der Feld- } \\
\text { forschung } \\
\text { (Primär- } \\
\text { material) }\end{array}$ & $\begin{array}{l}\text { Erkenntnis } \\
\text { zuwachsmit } \\
\text { definierten } \\
\text { Gültigkeits } \\
\text { umfang }\end{array}$ & $\begin{array}{l}\text { Schluss } \\
\text { folgerungen } \\
\text { für das } \\
\text { Treffen von } \\
\text { Ent- } \\
\text { scheidungen }\end{array}$ \\
\hline $\begin{array}{l}\text { Annahme }(n) \\
\text { treffen } \\
{[\text { Hypothese(n)] }}\end{array}$ & $\begin{array}{l}\text { Prüfen/ } \\
\text { Belegen }\end{array}$ & Belegen & $\begin{array}{l}\text { Behauptung(en) } \\
\text { formulieren } \\
\text { [These(n)] }\end{array}$ & Maßnahmen \\
\hline
\end{tabular}

Abb. 3: Etappen zum Gewinnen der Erkenntnisse 
bar. Sie beziehen sich beispielsweise auf Umsetzungsvorschläge, Leitungsentscheidungen, Wirtschaftlichkeitsbetrachtungen oder neue Forschungsfragen.

In $A b b .3$ ist der beschriebene Weg schematisch dargestellt. Das folgende Beispiel soll mit Bezug auf Abb. 3 das beschriebene Vorgehen veranschaulichen:

Die Forschungsfrage einer Graduierungsarbeit lautet:

Welcher Zusammenhang besteht zwischen der Kundenzufriedenheit und der Anzahl der Kundenbeschwerden?

Aus einem bestimmten Erfahrungsbereich entsteht als eine erste, vermutete, vorläufige Antwort auf die Forschungsfrage die folgende Annahme (wissenschaftliche Hypothese):

Wenn die Anzahl der Beschwerden gering ist, dann sind unsere Kunden zufrieden!

Nun folgt die Recherche in der wissenschaftlichen Literatur (Materialforschung). In ihrem Ergebnis liegen unterschiedliche Aussagen vor:

Eine Quelle A weist für einen definierten Untersuchungsbereich folgendes Ergebnis aus: Eine geringe Anzahl von Beschwerden ist Ausdruck von Kundenzufriedenheit.

In einer Quelle B wird für einen anderen Untersuchungsbereich festgestellt: Eine geringe Anzahl von Beschwerden ist kein Indikator für die Zufriedenheit der Kunden.

Die eingangs aufgestellte Hypothese ist offenbar nicht allgemein gültig. Jetzt ist mittels Feldforschung zu untersuchen, ob sie unter bestimmten Bedingungen verifiziert oder falsifiziert werden kann. Dafür wird das Instrument der schriftlichen Befragung eingesetzt. Aus einer Grundgesamtheit von 7300 haben 4000 Kunden von Unternehmen des Gebäudereinigungsgewerbes aus der Region X im Zeitraum 2005 bis 2011 auf die Frage nach ihrer Zufriedenheit mit dem Personal geantwortet. Das führt zu folgenden Ergebnissen:

$38 \%$ der befragten Kunden hatten diesbezüglich ein negatives Erlebnis, insbesondere ungenügende Beratung, schlechte Betreuung, Unfreundlichkeit des Personals.

Von den Kunden mit negativen Erlebnissen beschwerten sich $52 \%$. Mithin verzichteten $48 \%$ auf eine Beschwerde, aber nahezu alle aus diesem Kreis beabsichtigten, den Anbieter zu wechseln.

Von den Kunden, die Beschwerde geführt haben, waren 29 \% mit der entsprechenden Reaktion des Unternehmens zufrieden. $71 \%$ waren mit der Beschwerdebehandlung unzufrieden, aber nur ein sehr geringer Teil $(<20 \%)$ von ihnen äußerte eine Wechselabsicht.

Die wissenschaftliche Hypothese ist für den Bereich der Gebäudereinigung offenbar nicht haltbar, sie ist falsifiziert. Die Behauptung (These) lautet mit definiertem Gültigkeitsumfang:

Für Gebäudereinigungsunternehmen in der Region $\mathbf{X}$ war im Zeitraum 2005 bis 2011 eine geringe Anzahl von Beschwerden kein Indikator für Kundenzufriedenheit. Beschwerdeführende wanderten nur selten ab.
Die Schlussfolgerung für die Leitung der Gebäudereinigungsunternehmen lautet:

Animiere deine Kunden zur Beschwerde!

Eine sich anschließende Forschungsfrage könnte lauten: Wie ist die Beschwerde zu behandeln, damit der Kunde mit dem Ergebnis zufrieden ist?

\section{Sichern der Gültigkeit (Validität) der gewonnenen Erkenntnis und Aufzeigen einer Forschungsperspektive}

Die Grenzen der gewonnenen Erkenntnisse anzugeben, ihre Validität erkennbar zu kennzeichnen, gehört zu den Ansprüchen an eine wissenschaftliche Arbeit mit Prüfungscharakter. Im Verständnis des wissenschaftlichen Sprachgebrauchs wird Validität vor allem als Gütekriterien für Mess- und Testverfahren gebraucht. In diesem Text wird Validität im Sinne von Gültigkeit einer wissenschaftlichen Erkenntnis unter klar definierten Bedingungen verwendet. Für das Beispiel (Zusammenhang von Kundenzufriedenheit und Anzahl der Beschwerden) kann die Untersuchung unter den definierten Bedingungen als intern valide bezeichnet werden. Die gewonnene Erkenntnis erlaubt eine eindeutige Schlussfolgerung in Hinsicht auf die kausale Beeinflussung der abhängigen Variablen (Kundenzufriedenheit) durch die unabhängige Variable (Anzahl der Beschwerden) (vgl. Eid et al. 2017, S. 85).

Auf dem beschriebenen Wege zur Erkenntnis ergeben sich mitunter ergänzende oder gänzlich neue Aspekte, angeregt durch das Literaturstudium oder Fachgespräche. Die Versuchung ist da, diese aufzunehmen, allerdings häufig mit dem Ergebnis, dass die „Bugwelle“ des inhaltlichen Bewältigens immer höher wird und die verfügbare Zeit immer knapper. Deshalb gilt der Rat, die eingangs gestellte Forschungsfrage konsequent zu beantworten, beim Nachweis des angenommenen Zusammenhangs zu bleiben (vgl. Theisen, 2017, S. 50). Allerdings ist das gewonnene Ergebnis dann mit Hinweis auf seine Gültigkeit einzuschränken. Die Schlussbetrachtungen weisen dann u. a. auf jene anderen Aspekte hin, die das Ergebnis beeinflussen könnten und deshalb künftig untersucht werden sollten. Mit dem Erschließen von weiteren Themen im Kontext der eigenen Untersuchung eröffnet der Autor eine Forschungsperspektive und leistet einen Beitrag für die Wissenschaftsentwicklung.

Das Schema in Abb. 4 soll durch das folgende Beispiel (in Anlehnung an Ebster, Stalzer, 2003, S. 98 ff.) illustriert werden:

Annahme (Hypothese): Es gibt einen Zusammenhang zwischen Kundenzufriedenheit und Schnelligkeit der Schadensregulierung durch die Kfz-Versicherung.

Beweis: So zeigt eine Untersuchung von Müller (2000, S. 25 ff.), dass Kunden einer Autoversicherung, die im 


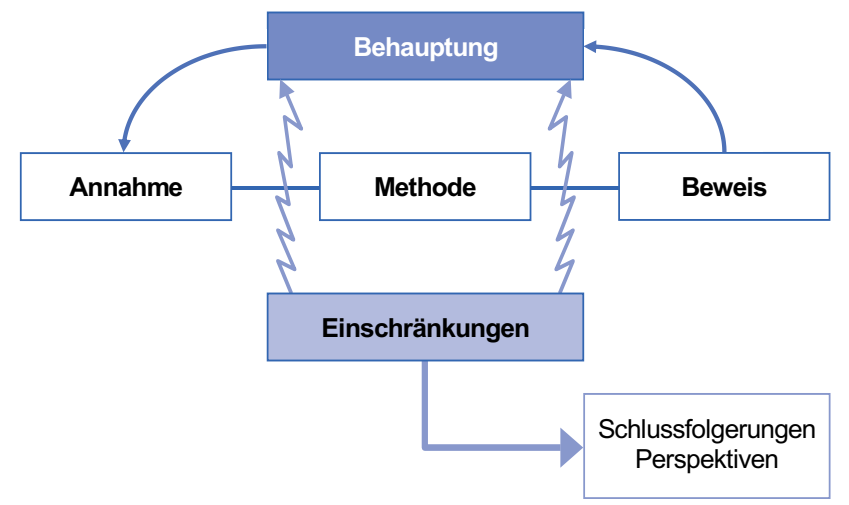

Abb. 4: Schema der Einschränkung wissenschaftlicher Ergebnisse

Schadensfall länger als zwei Wochen auf die Begleichung ihres Schadens warten mussten, im folgenden Jahr doppelt so häufig die Versicherung wechselten als Kunden, bei denen die Versicherung schneller reagierte.

Behauptung (These): Die Zufriedenheit der Kunden mit ihrer Kfz-Versicherung ist abhängig von der Schnelligkeit der Schadensregulierung.

Einschränkung: Allerdings ist aus der Studie von Mauer (1998, S. 49 f.) in der Hausratversicherung ersichtlich, dass die Erreichbarkeit des Ansprechpartners im Unternehmen und die Transparenz der Schadensabwicklung für den Versicherungskunden annährend gleiche Bedeutung haben wie die Schnelligkeit der Schadensregulierung.

Schlussfolgerung: Demzufolge ist die eingangs getroffene Behauptung wie folgt zu präzisieren: Die Zufriedenheit von Kunden mit ihrer Kfz-Versicherung ist neben anderen Faktoren auch abhängig von der Schnelligkeit der Schadensregulierung.

Perspektive: In künftigen Untersuchungen zur Kundenzufriedenheit mit Kfz-Versicherungen sind des weiterem die
Transparenz der Abwicklung und die Erreichbarkeit des Ansprechpartners einzubeziehen.

Hinweis: Quellen im oben genannten Beispiel sind fiktiv!

\section{Schlussbemerkung}

Die Erweiterung des Erkenntnisstandes, so wurde eingangs postuliert, ist ein angestrebtes Ergebnis von Bachelor- und Masterarbeiten. Im Kontext dieser Arbeiten ist sie ein wesentliches Mittel zum Zweck, der im Nachweis einer Befähigung zum wissenschaftlichen Arbeiten besteht. Diese Befähigung findet ihren Ausdruck im Finden einer bearbeitbaren Aufgabenstellung, dem Beschreiten eines nachvollziehbaren Weges zur Erkenntnis, dem Gültigkeitsnachweis der Ergebnisse und dem Aufzeigen einer Forschungsperspektive. Dazu gehört auch das Einhalten der Konventionen wissenschaftlichen Arbeitens, wie Zitieren, Dokumentieren, Verzeichnen, Textgestalten etc. Diese werden in den meisten Fällen durch die betreffende Hochschule mit Besonderheiten geregelt.

\section{Literatur}

Ebster, C.; Stalzer, L.: Wissenschaftliches Arbeiten für Wirtschafts- und Sozialwissenschaftler. 2., überarb. Aufl., Wien 2003.

Eid, M.; Gollwitzer, M.; Schmitt, M.:. Statistik und Forschungsmethoden. 5., korrigierte Aufl., Weinheim Basel: Beltz 2017.

Karmasin, M.; Ribing, R.: Die Gestaltung wissenschaftlicher Arbeiten. 7., aktualisierte Aufl., Wien 2013.

Lehmann, G.: Wissenschaftliche Arbeiten zielwirksam verfassen und Präsentieren. 6., erweiterte Aufl., Renningen 2017.

Rettig, H.: Wissenschaftliche Arbeiten schreiben. Stuttgart 2017.

Theisen, M. R.: Wissenschaftliches Arbeiten. Technik - Methodik - Form. 17., aktualisierte und bearbeitete Aufl., München 2013.

Trimmel, M.: Wissenschaftliche Arbeiten in der Psychologie und Medizin. Wien 2009. 